\title{
Layer topology on vertex chain simplicial complexes
}

\author{
Anas $\mathrm{E}^{1 *}$ and Baby Chacko ${ }^{2}$
}

\begin{abstract}
In order to study the topological properties of complex shapes, it is convenient to view them as the union of simplex gluing together in a specific manner to form simplicial complexes. Simplex are the simple geometric objects like points, line segments, triangles, tetrahedra etc. Layer Topology on simplicial complexes helps us to understand the topological properties of complex shapes by comparing the topological properties of closed unit interval in the usual topology.
\end{abstract}

Keywords

Simplicial complex; Barycentric maps; Layer Topology.

\section{AMS Subject Classification}

$55 \mathrm{U} 10,55 \mathrm{U} 15$.

${ }^{1}$ Department of Mathematics, MES Mampad College, Kerala-676542, India.

${ }^{2}$ Department of Mathematics, St.Joseph's College, Devagiri, Calicut, Kerala, India.

*Corresponding author: ${ }^{1}$ anas.edarath@gmail.com; ${ }^{2}$ babychacko12@gmail.com

Article History: Received 24 January 2019; Accepted 24 May 2019

(C)2019 MJM.

\section{Contents}

1 Introduction .490

2 Types of Simplicial Complexes 490

3

Pasting Lemma .491

4 Layer Topology on Vertex Chain Complex. .491

5

References 492

\section{References...}

\section{Introduction}

\section{Barycentric Maps and Layer Topology}

Let $a_{0}, a_{1}, \ldots a_{n}$ be geometrically independent set in $R^{N}$. We define the n-simplex $\sigma$ spanned by $a_{0}, a_{1}, \ldots a_{n}$ be the set of all points $\mathrm{x}$ of $R^{N}$ such that $x=\sum_{i=0}^{n} t_{i} a_{i}$, where $\sum_{i=0}^{n} t_{i}=1$ and $t_{i} \geq 0$ for all $i$. The numbers $t_{i}$ are uniquely determined by $x$; they are called the barycentric coordinates of the point $x$ of $\sigma$ with respect to $a_{0}, a_{1}, \ldots a_{n}$.

If $x$ is a point of the polyhedron $|K|$, then $x$ is the point of one simplex $\mathrm{K}$ whose vertices are $a_{0}, a_{1}, \ldots a_{n}$.Then $x=\sum_{i=0}^{n} t_{i} a_{i}$, where $\sum_{i=0}^{n} t_{i}=1$ and $t_{i} \geq 0$ for all $i$. If $\mathrm{v}$ is an arbitrary vertex of $\mathrm{K}$, then the barycentric coordinates $t_{v}(x)$ of $x$ with respect to $v$ is $t_{v}(x)=0$ if $v \neq a_{i}$ and $t_{v}(x)=t_{i}$, if $v=a_{i}$.

By [1], Let $v=a_{i}$ be any vertex of a simplex $\sigma$ of dimension $n \geq 1$ with vertices $a_{0}, a_{1}, \ldots a_{n}$. Then $t_{v}\left(a_{j}\right)=1$ for $i=j$ and $t_{v}\left(a_{j}\right)=0$ for $i \neq j$.

Let $v=a_{0}$ be a vertex of a simplex $\sigma$ of dimension $n \geq 1$ with vertices $a_{0}, a_{1}, \ldots a_{n}$, then the map $t_{v}: \sigma \rightarrow[0,1]$ is called barycentric map with respect to $\mathrm{v}$.

Let $v=a_{0}$ be a vertex of a simplex $\sigma$ of dimension $n \geq 1$ with vertices $a_{0}, a_{1}, \ldots a_{n}$ and let $t \in[0,1]$, then the set $l_{t}(\sigma)=\left\{x \in \sigma: t_{v}(x)=t\right\}$ is called the $t^{t h}$ layer of $\sigma$.

Let $\mathrm{U}$ be a subset of $I=[0,1]$, then $l_{U}=\left\{l_{t}: t \in U\right\}$. Whenever $U$ is open we can call $l_{U}$ as open layer.

The collection $\mathrm{L}$ of open layers $l_{U}$ form a basis for a topology on $\sigma$

Let $v=a_{0}$ be a vertex of a simplex $\sigma$ of dimension $n \geq 1$ with vertices $a_{0}, a_{1}, \ldots a_{n}$. Then the topology on $\sigma$ in which open layers form a basis is called layer topology.

\section{Types of Simplicial Complexes}

A Simplicial Complex on $\mathrm{Rm}$ is a set $\mathrm{K}$ consisting of a finite or infinite set of simplices in $\mathrm{Rm}$ having the following conditions

1. Every face of a simplex is also belongs to $\mathrm{K}$

2. For any two simplex $\sigma_{1}$ and $\sigma_{2}$ in $\mathrm{K}$, If $\sigma_{1} \cap \sigma_{2} \neq \Phi$, then $\sigma_{1} \cap \sigma_{2}$ is a common face of both $\sigma_{1}$ and $\sigma_{2}$ 
or

For any two distinct simplex $\sigma_{1}$ and $\sigma_{2}$, int $\sigma_{1} \cap \sigma_{2}=\phi$,

Every $\mathrm{k}$-simplex in $\mathrm{K}$ is called a $\mathrm{k}$-face of $\mathrm{K}$. Zero face is called a vertex and one face is called an edge.

The dimension of a simplicial complex $\mathrm{K}$ is the maximum of the dimension of all simplices in $\mathrm{K}$. If $\operatorname{dim} K=d$, then every face of dimension $d$ is called a cell and every face of dimension $d-1$ is called a facet.

The union $|K|$ of all simplices in $\mathrm{K}$ is a subset of $R^{m}$. We can define a topology on $|K|$ by defining a subset $\mathrm{F}$ of $|K|$ to be closed if and only if $F \cap \sigma$ is closed in $\sigma$ for every face $\sigma \in K$. The resultant topological space is called the geometric realization of $\mathrm{K}$.

For any simplex $\sigma$, we have $|\sigma|=\sigma$.

A polytope is the geometric realization of some simplicial complex. A polytop of dimension 1 is usually called a polygon and a polytop of dimension 2 is called polyhedron.

When $\mathrm{K}$ consists of infinitely many simplices, we usually require that $\mathrm{K}$ be locally finite, which means that every vertex belongs to finitely many faces. If $\mathrm{K}$ is locally finite, then its geometric realization $|K|$ is locally compact. In this paper, we will consider only finite simplicial complexes, that is the complex having finite number of simplices. In finite simplicial complex, the topology of $|K|$ defined above is same as the topology induced from $R^{m}$. In this case, for any simplex $\sigma$ in $\mathrm{K}$, Int $\sigma$ coincide with interior $\sigma^{0}$ of $\sigma$ in topological sense and the boundary $\partial \sigma$ coincide with boundary of $\sigma$ in the topological sense.

Let $K_{2}$ be a complex and let $K_{1} \subseteq K_{2}$ Then $K_{1}$ is said to be a subcomplex of $K_{2}$ if and only if $K_{1}$ is a complex.

For any complex $\mathrm{K}$ of dimension $\mathrm{d}$, for any $i$ with $0 \leqslant i \leqslant d$, the subset $K^{(i)}=\{\sigma \in K: \operatorname{dim} \sigma \leq i\}$ is called the $i$ - skeleton of $\mathrm{K}$. then $K^{i}$ is a subcomplex of $\mathrm{K}$.

$K^{i}=\{\sigma \in K / \operatorname{dim} \sigma=i\}$ is not a complex. $K^{0}$ is the set of all vertices of $\mathrm{K}$. A simplicial complex $K_{1}$ is a subdivision of a complex $K_{2}$ if and only if $\left|K_{1}\right|=\left|K_{2}\right|$ and if every face of $K_{1}$ is a subset of some face $K_{2}$. A complex $\mathrm{K}$ of dimension $\mathrm{d}$ is pure(or homogeneous) if and only if every face of $\mathrm{K}$ is some face of some d-simplex(or some cell $\mathrm{K}$ ).

A Complex is connected if and only if $|K|$ is connected.

Let $\mathrm{K}$ be any complex and $\sigma$ be any face of K. Then star of $\sigma$ denoted by $S t(\sigma)$ or $S t(\sigma, K)$ is the subcomplex of K consisting of all faces $\tau$ containing $\sigma$ and all faces of $\tau$.

St $(\sigma)=\{s \in K: \exists \tau \in K$ such that $\sigma \subseteq \tau$ and $s \subseteq \tau\}$

The link of $\sigma$ denoted by $\operatorname{Lk}(\sigma)$ or $\operatorname{Lk}(\sigma, K)$ is the subcomplex of K consisting of all faces in $\operatorname{St}(\sigma)$ and do not intersect $\sigma$.

$L k(\sigma)=\{\tau \in K: \tau \in S t(\sigma)$ and $\sigma \cap \tau=\phi\}$

If $\mathrm{K}$ is a pure complex of dimension $\mathrm{d}$, then star of $\sigma$ is also pure with dimension d. If dimension of $\sigma$ is $\mathrm{k}$ then $\operatorname{Lk}(\sigma)$ is pure of dimension $d-k-1$.

Open star of $\sigma, s t(\sigma)$, is defined as the subspace of $|K|$ con- sists of interiors of all faces $\tau$ containing $\sigma$.

$s t(\sigma)=\{\tau-\partial \tau: \sigma \subseteq \tau$ and $\tau \in K\}$

Open star is not a complex, but it is a subset of $|K|$.

$$
\overline{s t(\sigma)}=|s t(\sigma)|
$$

If $\sigma$ is a vertex, we have $l k(v)=\overline{s t(v)-s t(v)}$

$\operatorname{st}(\sigma)$ is open for all $\sigma$.

For any point $a \in|K|$, there is a unique smallest simplex $\sigma=\left(a_{0}, a_{1}, \ldots . a_{k}\right)$ such that $a \in \operatorname{int}(\sigma)$

ie $=\sum_{i=0}^{n} t_{i} a_{i}$, where $\sum_{i=0}^{n} t_{i}=1$

and $t_{i} \geq 0$ for all $i$ with $0 \leq i \leq k$.When $k=0, t_{0}=1$ and $a_{0}=a$.

Hence $\operatorname{st}(v)=a \in|K|: t_{v}(a)>0$

Thus $|K|-s t(v)$ is the union of all faces of $\mathrm{K}$ that do not contain $\mathrm{v}$ as a vertex. Then clearly it is a closed set. Thus $s t(v)$ is open in $|K|$. st $(v)$ is path connected also.

\section{Pasting Lemma}

Let $\mathrm{A}$ and $\mathrm{B}$ be closed subsets of a topological space $\mathrm{X}$ and $f: A \rightarrow Y$ and $g: B \rightarrow Y$ are continuous functions such that $f(x)=g(x)$ for all $x \in A \cap B$. Then there exist a unique continuous map from $A \cup B$ to $\mathrm{Y}$ whose restriction to $\mathrm{A}$ is $\mathrm{f}$ and to $\mathrm{B}$ is $\mathrm{g}$. The result can be extended to finitely many closed sets which cover $\mathrm{X}$.

Definition 3.1. A simplex $\sigma \in K$ is said to be a component simplex of $K$ if there is no simplex $\tau \in K$ such that $\sigma$ is a proper face of $\tau$. That is $\sigma$ is a component simplex if $\sigma$ is a maximal simplex in $K$ containing all faces of $\sigma$.

Definition 3.2. A simplicial complex in which exactly one vertex in each component simplex (except the first one), is a vertex on the facet of the preceding component simplex is called a vertex chain complex.

\section{Layer Topology on Vertex Chain Complex}

Let $\mathrm{S}$ be a vertex chain simplicial complex with components $\sigma_{1}, \sigma_{2}, \ldots, \sigma_{n}$ (taken in order).

Let $\sigma_{k}=\left(a_{k_{1}}, a_{k_{2}}, \ldots ., a_{k_{m_{k}}}\right)$ be the $k^{\text {th }}$ simplex and $a_{k_{1}}$ be the vertex common to $\sigma_{k}$ and $\sigma_{k-1}$ for all $k \neq 1$. Suppose the Layer topology is defined on each $\sigma_{k}$ with $a_{k_{1}}$ as the fixed vertex to define the corresponding barycentric map.

Let $s_{a_{k_{1}}}: \sigma_{k} \rightarrow[0,1]$ be the barycentric map with respect to $a_{k_{1}}$, and let $s:[0,1] \rightarrow[0,1]$ be defined by $s(t)=1-t$ . Define $t_{a_{k_{1}}}: \sigma_{k} \rightarrow[0,1]$ by $t_{a_{k_{1}}}=\operatorname{sos}_{a_{k_{1}}}$ then we can define layer topology on $\sigma_{k}$ by keeping open layers as a basis. $\left(t_{a_{k_{1}}}\right)^{-1}:[0,1] \rightarrow \sigma_{k}$ is continuous and open by considering $\sigma_{k}=\bigcup_{t \in I} l_{t}\left(\sigma_{k}\right)$ where $l_{t}\left(\sigma_{k}\right)=\left\{x \in \sigma: t_{a_{k_{1}}}(x)=t\right\}$

Define $f_{k}: I_{k}=[(k-1) / n, k / n] \rightarrow[0,1]$ by $f_{k}(t)=n t-k+1$, as it is a polynomial function $f_{k}$ is continuous. Also, we can see that $f_{k}$ is bijective.

Let $g_{k}:[(k-1) / n, k / n] \rightarrow \sigma_{k}$ be defined by $\left.g_{k}(t)=\left(\left(t_{a_{k_{1}}}\right)\right)^{-1} o f_{k}\right)(t)$. 
Since $\left(t_{a_{k_{1}}}\right)^{-1}$ and $f_{k}$ are continuous and open, $g_{k}$ is also continuous and open.

$$
\begin{gathered}
\text { Consider } g_{k}:[(k-1) / n, k / n] \rightarrow \sigma_{k} \text { and } \\
g_{k+1}:[k / n,(k+1) / n] \rightarrow \sigma_{k+1}
\end{gathered}
$$

By definition $g_{k}(k / n)$ on $\sigma_{k}$ and $g_{k+1}\left(k / n\right.$ on $\sigma_{k+1}$ are coincide. By pasting lemma the function $f:[0,1] \rightarrow S$ where $f$ restricted to $[(k-1) / n, k / n]$ is $g_{k}$ and it is continuous.

As in [1], we can form layers on vertex chain complex $S$ which will form a basis for a topology on $\mathrm{S}$ and we can define it as Layer topology on S.

As per [2], [0,1] and a simplex $\mathrm{S}$ are homeomorphic with respect to Layer Topology. Constituting all results together we can conclude that a simplex $\sigma$ and a vertex chain complex $\mathrm{S}$ are homeomorphic.

\section{Conclusion}

Simplex of any dimension is homeomorphic to any common vertex simplicial complexes with respect to the layer topology defined on both.

\section{References}

[1] Anas E and Baby Chacko, Barycentric Map and Layer Topology on Simplices, International Journal of Mathematics Trends and Technology (IJMTT), X(2017), 33-35

[2] Anas and Baby Chacko, Persistence of Toplogical Properties through Layer Map, International Journal of Mathematics Trends and Technology(IJMTT),(ISSN: 22315373)59(2018), 85-87

[3] James R. Munkers, Elements of Algebraic Topology, Addison-Wesley Publishing Company, 1984.

[4] K.D.Joshi, Introduction to General Topology,New Age International (P) Ltd., Publishers, 2006.

[5] J. Munkres, Topology, Prentice-Hall, Inc. Englewood Cliffs, N.J. 1975.

[6] John B Fraleigh, A First Course in Abstract Algebra,Dorling Kindersley (India) Pvt Ltd., 2006.

[7] Tamal K Dey, Herbert Edelsbrunner, Sumanta Guha and Dmitry V. Nekhayev, Topology Preserving Edge Contraction,Publication De L'Institute Mathematique,66(1999), 23-45

[8] Herbert Edelsbrunner and Nimish R. Shah, Triangulating Topological Spaces,International Journal of Computational Geometry and Applications, 7(1997), 365-378

[9] D.Burghelea and T.K.Dey, Topological persistence for circle valued maps, Discrete Comput. Geom., 50(2013), 69-98.

[10] O. Busaryev, S.Cabello, C.Chen, T.K.Dey and Y wang, Annotating simplices with homology basis and its ap-
plications.Proc. 13th Scandinavian Sympos. Workshops algorithm Theory(SWAT 2012),1(2012), 189-200.

[11] G.Calsson and V. de Silva, Zigzag persistence, Fond. Comput. Math., 10(2010), 367-370.

[12] G.Calsson, V. de Silva and D. Morozov, Zigzag persistent homology and real- valued functions, Proc. 26thAnnu. Sympos. Comput. Geom. (2009), 247-256.

[13] Richard jerrard, Homology with Multiple-valued Functions Applied to Fixed Points. Transactions of The American Mathematical Society, 213(1975), 1-10.

[14] Allen Hatcher, Algebraic Topology, Cambridge University Press(2002).

[15] Raymond Earl Smithson, Some General Properties Of Multi-Valued Functions,Pacific Journal of Mathematics, 15(1965), 1-8.

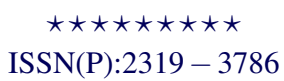

Malaya Journal of Matematik

$\operatorname{ISSN}(\mathrm{O}): 2321-5666$

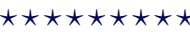

